\title{
Interactive Hyperspectral Image Visualization Using Convex Optimization
}

\author{
Ming Cui, Anshuman Razdan, Member, IEEE,, Jiuxiang Hu, and Peter Wonka, Member, IEEE,
}

\begin{abstract}
In this paper we propose a new framework to visualize hyperspectral images. We present three goals for such a visualization: preservation of spectral distances, discriminability of pixels with different spectral signatures, and interactive visualization for analysis. The introduced method considers all three goals at the same time and produces higher quality output than existing methods. The technical contribution of our mapping is to derive a simplified convex optimization from a complex nonlinear optimization problem. During interactive visualization, we can map the spectral signature of pixels to RGB colors using a combination of principal component analysis and linear programming. In the results we present a quantitative analysis to demonstrate the favorable attributes of our algorithm.
\end{abstract}

Index Terms-hyperspectral image visualization, perceptual color distances, PCA, linear programming.

\section{INTRODUCTION}

Hyperspectral images contain hundreds of spectral samples per pixel. To visualize such images, the many spectral bands must first be projected to a lower dimensional space, typically the RGB color space of a monitor.

In this paper we present a framework to visualize hyperspectral images. The problem can be formulated as follows. A $w \times h$ hyperspectral image with $\mathrm{d}$ bands can be seen as a higher dimensional tensor $T_{d} \in R^{w \times h \times d}$ where each of the $w h$ pixels is described by a vector $X_{i}$ with $d$ spectral samples. As output of this algorithm we want to map the image to a lower dimensional display range, i.e. a tensor $T_{k} \in R^{w \times h \times k}$ with all entries constrained to lie between 0 and 1 (normalized display range) and $k<d$. In this paper, we consider the case $k=3$ to map to the display range of a color monitor.

We set out by defining three goals for such a visualization: 1) The distances from the input spectral space should be preserved in the output color space to provide a perceptually meaningful visualization, 2) the algorithm should make use of the dynamic range of the display device to show details in the image, and 3) the algorithm should allow for interactive exploration.

Hyperspectral image visualization is usually provided as a functionality in hyperspectral image analysis software such as Multispec [1], ENVI [2], Geomatics [3], TnTlite [4], HyperCube [5] and HIAT [6]. A direct visualization method is to render the image as a 3D cube [3], [6]. To explore different bands as grayscale images, one set of tools allows a user to cycle through all bands or to flicker between two bands [5]. To extract an RGB color image for visualization, interactive tools can be used to pick three bands and assign them to the red, green, and blue channels directly [2], [1]. More sophisticated mappings can be created through user-specified linear combinations of spectral bands [5], data independent visually meaningful linear combinations [7], or data dependent automatically computed combinations using PCA (Principle Component Analysis) or MNF (a noise reduction version of PCA) [2], [4]. Additionally, ICA (Independent Component Analysis) has been proposed for dimension reduction [8], [9], but ICA is significantly slower than PCA and it is not clear how to rank the significance of different channels provided by ICA. An alternative idea for visualization would be to borrow from non-linear methods for dimension reduction, such as local linear embedding(LLE) and ISOMAP. Two recent nonlinear color mapping approaches by Gooch et al. [10] and Rasche et al. [11] report running times of minutes to compute a mapping for input images with three spectral samples. In general, we expect these existing non-linear techniques to require significantly more computation time than linear methods, especially for $d>>3$.

The main problem that we observed is that existing methods map spectral samples to unbounded three-dimensional Euclidian space. After dimension reduction they all use a second nonuniform mapping to color space that creates colorful images but also the illusion of salient features that are not present in the data. Examples are non-uniform scaling, standard deviation stretch, and histogram equalization. Therefore, these algorithms sacrifice the first goal (preservation of spectral distances) to satisfy the second (using the dynamic range of the display).

In this paper we propose a novel strategy for hyperspectral image visualization that uses a higher quality mapping. The main idea of our approach is to derive a fast optimization procedure that can perform dimension reduction while considering the boundaries of the HSV [12] color space (see figure 4 for a short introduction). During the visualization a user can interactively explore the hyperspectral dataset using spatial and spectral lenses to configure a non-linear mapping. Our major contribution is as follows:

- We present a high quality framework for hyperspectral image visualization. We provide the quality of non-linear methods, while preserving much of the interactivity only available with simple linear methods. Both visual and quantitative comparisons suggest that our method satisfies the three goals simultaneously better than existing methods.

\section{RELATED WORK}

We review related work in three categories: hyperspectral image visualization, color to gray mapping, and dimensionality reduction. 


\section{A. Hyperspectral Image Visualization}

Traditionally, hyperspectral images have been visualized as a cube with a suite of interactive tools [13]. One set of tools allows a user to extract one spectral band at a time or cycle through spectral bands as an animation. To create RGB images, interactive tools can be used to specify red, green, and blue values as linear combinations of spectral bands. This means an RGB value is computed by a matrix vector multiplication. Along these lines several authors have suggested methods to automatically create linear combinations of spectral bands to define the green, red, and blue color channels of a visualization [14], [15], [7], [16]. In this paper we compare our results to two such methods: 1) The Color Matching Function(CMF) algorithm proposed by Jacobson et al. [7], and 2) a traditional PCA based method described in [14], Section II. The main problem in existing visualization software toolkits is the application of post processing algorithms to enhance the image. Examples are non-uniform scaling, standard deviation stretch, and histogram equalization. These algorithms disproportionately enhance minor features. It is worth noting that a visualization can be specifically designed for different applications. For example, a visualization can be used as a post-process for classification [17].

\section{B. Color to Gray Mapping}

In recent years, transforming color images to gray scale attracted the interest of several researchers [10], [18], [11], [19]. The problem is to find a lower dimension embedding of the original data that can best preserve the contrast between the data points in the original data. While these papers are an inspiration for our work, their methodologies do not easily extend to higher dimensions, due to memory consumption and computation time.

\section{Dimensionality Reduction}

There is a larger number of general dimensionality reduction algorithms in the literature. Prominent examples are ISOMAP [20], Local Linear Embedding [21], Laplacian Eigenmap Embedding [22], Hessian Eigenmap Embedding [23], Conformal Maps [24], and Diffusion Maps [25]. These algorithms are theoretically very strong. However, there are two issues. First, these algorithms assume that the data lies in a nonlinear submanifold in the original space. This assumption must be verified before these nonlinear dimension reduction methods can be used on hyperspectral images. Although previous work [26], [27] suggests that nonlinearity exists in hyperspectral imagery, the nonlinearity is typically data-dependent. Second, nonlinear dimension reduction methods are usually slow and memory intensive. For example, a small $100 \times 100$ image gives rise to a distance matrix with $100^{4}=100$ million entries. Computing the SVD decomposition (a typical step needed in these methods) does not scale well to larger images and a $500 \times 500$ image is already out of reach for current workstations. In [28], an accelerated version of ISOMAP is implemented for hyperspectral images. The method greatly enhances the algorithm speed but running times are still not fast enough for an interactive visualization.

\section{OVERVIEW}

Here we give an overview of the paper. First, we lay out three goals of the visualization and derive quantitative metrics that we will use to compare our algorithm to previous work. Second, we give the motivation for our algorithm and explain how we derived it. Third, we give a short description of the individual steps of the algorithm.

\section{A. Goals}

Preservation of distances: The first goal of our visualization is to create an image such that the perceptual color distances are similar to the Euclidian distances between the high dimensional spectral samples. We follow the argumentation which suggests that the Euclidian distance in RGB color space is not a good measure for the perceptual distance (see for example [29] and [14]). Therefore, we attempt to preserve Euclidian distances in a perceptual colors space, $\mathrm{L}^{*} \mathrm{a}^{*} \mathrm{~b}^{*}$ [12]. To evaluate the preservation of distances in $\mathrm{L}^{*} \mathrm{a} * \mathrm{~b} *$ we define a correlation based metric similar to [7]. Let $X$ be the vector of all pairwise Euclidian distances of the pixels in the high dimensional spectral space and let vector $Y$ be the corresponding pairwise Euclidean distances of the pixels in $\mathrm{L}^{*} \mathrm{a} \mathrm{b}^{*}$ space. The correlation $\gamma$ can be calculated using the following formula:

$$
\gamma=\frac{X^{T} Y /|X|-\bar{X} \bar{Y}}{s t d(X) \cdot \operatorname{std}(Y)}
$$

$|X|$ denotes the number of elements in $X, \bar{X}$ and $\operatorname{std}(X)$ denote the mean and standard deviation respectively. In the ideal case the normalized correlation equals 1 and the closer the correlation is to 1 the better the distance is preserved. In practice, the images we consider are too large to consider all pairwise distances, so that we accelerate the computation by subsampling.

It is important to discuss alternative design choices for metric $\gamma$. An alternative to Euclidian distances in original space is to only consider the spectral angles in original space, e.g. [7]. While spectral angles contain enough information for endmember identification in classification applications, the radiant intensities in original space carry additional information about ground textures that reflect shape and contours [7]. This is very important for a series of tasks such as georeferencing [2], registration [30] and change detection [31]. Another idea suggested by [7] is to ignore the luminance values in $\mathrm{L}^{*} \mathrm{a} \mathrm{b}^{*}$ space. In our experience, better performance can be achieved when making use of the full color space including the luminance value.

Separability of features: Correlation alone does not guarantee that colors can be well distinguished. It is still possible that the resulting image is too dark or too bright, because the color space is not efficiently used and too many pixels fall within a smaller part of the color space. Therefore, we use a metric $\delta$ that measures how well pixels are mapped to distinguishable colors. The key idea is that the average distance between two pixels in perceptual color space should be as large as possible: 


$$
\delta=|Y|_{1} /|Y|
$$

where $Y$ is from equation $1,|Y|_{1}$ denotes the L1 norm, and $|Y|$ is the number of elements of the vector. Therefore $\delta$ denotes the average pairwise Euclidean distance in the $\mathrm{L}^{*} \mathrm{a}^{*} \mathrm{~b} *$ color space. The same metric was independently suggested by [16]. Larger values of $\delta$ indicate a better separability of features and we therefore try to maximize $\delta$.

Interactive visualization: For an effective visualization the data set should be interactively explored by a human user. We consider two aspects of the interactive visualization. First, the computation time should not exceed a few seconds. Second, it is important to have a method that is compatible with interactive tools for data exploration. In this paper we introduce spatial and spectral lenses as example tools and show how the algorithm can be integrated into an interactive hyperspectral image visualization framework.

\section{B. Design Choices}
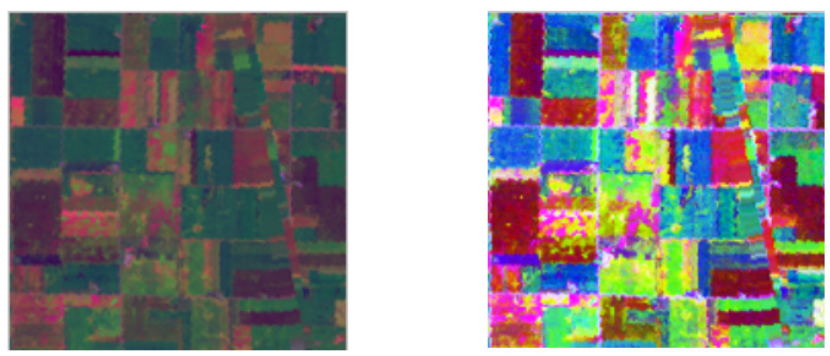

Fig. 1. Comparing two visualization algorithms for a multi spectral data set. Left: visualization with PCA. Right: visualization with enhanced PCA. Note how smaller features are exaggerated. The first three eigenvectors are mapped to $(R, G, B)$ channels respectively.

We first present our analysis of the state of the art and then discuss three possible approaches to improve the visualization. The third approach is the one we follow in this paper.

State of the Art: State-of-the-art techniques use a two-step framework which is shown in the first row of figure 2. These techniques map the high dimensional spectral samples to an unbounded 3D Euclidean Space in the dimension reduction step. Even though the mathematical transformations, such as PCA and ICA, perform typically well when mapping to an infinite space, the color space has an actual boundary that needs to be respected. We call this problem the boundary problem. Please note that this problem is similar to the tone reproduction problem in computer graphics [32]. For example, the RGB color space has a cube as a boundary and the Lab color space is bounded by concave surfaces. Therefore, these methods need a second transformation which actually maps 3D points to RGB triples for visualization purposes. The simplest transformation is uniformly scaling the point set so that it fits into a cube. The three coordinates of the scaled space can be used as the red, green, and blue color values. Usually the points are sparsely distributed in the cube so that the resulting image tends to be too dark (see figure 1 left). In the quantitative analysis this visualization has good preservation of distances $(\gamma)$, but not a high separability of features $(\delta)$. Therefore, different alternatives to uniform scaling have been proposed to enhance the separability of features $(\delta)$ of the final image such as an exponential transform, non-uniform scaling, standard deviation mapping, the auto normalization transform, histogram equalization [33] and tone mapping [34] techniques. An example of enhanced PCA visualization is shown in figure 1 right. However, these techniques work on each color channel separately and therefore distort perceptual color distances non-uniformly. As a result minor features in the data can be disproportionably exaggerated and the preservation of distances is poor $(\gamma)$.

Approach One: The first idea we considered was to replace the second mapping with a mapping to $\mathrm{L}^{*} \mathrm{a} * \mathrm{~b} *$ color space to better keep perceptual distances (see figure 2, row 2). The $\mathrm{L}^{*} \mathrm{a}^{*} \mathrm{~b} *$ color space is the most popular choice in this context. However, the envelope of $\mathrm{L}^{*} \mathrm{a}^{*} \mathrm{~b}^{*}$ space has a concave and curved boundary and the cascading of two mappings produced similar undesirable side effects to existing methods.

Approach Two: To avoid complications due to two mappings, we intended to directly map points in $E^{d}$ to $\mathrm{L}^{*} \mathrm{a}^{*} \mathrm{~b}^{*}$ space, posing the problem as a constrained optimization (see figure 2, row 3).

Approach Three: Approach two seems to be the ideal solution for preserving perceptual distance. However, the $\mathrm{L}^{*} \mathrm{a} * \mathrm{~b} *$ color space has concave non-linear boundaries. A constrained optimization that maps the dataset into $\mathrm{L}^{*} \mathrm{a}^{*} \mathrm{~b} *$ is very time consuming and does not meet our interactive speed requirement. Therefore, we opted for a third approach (see figure 2, row 4) that uses the HSV color space instead of $\mathrm{L}^{*} \mathrm{a}^{*} \mathrm{~b}^{*}$ color space. Although HSV color space is not as good as $\mathrm{L}^{*} \mathrm{a} \mathrm{b}^{*}$ space, it is still much better than RGB color space in preserving perceptual distance. Our experiments will show that even though we optimize color distances in the HSV color space, the distances in the $\mathrm{L}^{*} \mathrm{a}^{*} \mathrm{~b}^{*}$ color space are well preserved according to our correlation metric. Additionally, this simplification allowed us to derive a solution based on a convex optimization that can be solved at interactive speeds. Overall, using HSV color space is a good compromise between quality and speed requirements. Next, we present an overview of our method and fill in algorithm details in section IV.

\section{Pipeline}

The proposed framework includes four steps (see figure. 3) described in the following.

1) Preprocessing: The motivation for pre-processing is to accelerate the computation time. Pre-processing consists of a vector quantization method to cluster the spectral signature of image pixels into $M$ clusters. Optimization is performed on cluster representatives and interpolation is performed on the remaining spectral samples. We implemented the faster median cut algorithm [35] and the higher quality k-means algorithm [36]. For each cluster, we select one representative point. The output of this stage are the representative points and cluster membership information for all the pixels (spectral samples). See section IV-A for details. The performance 


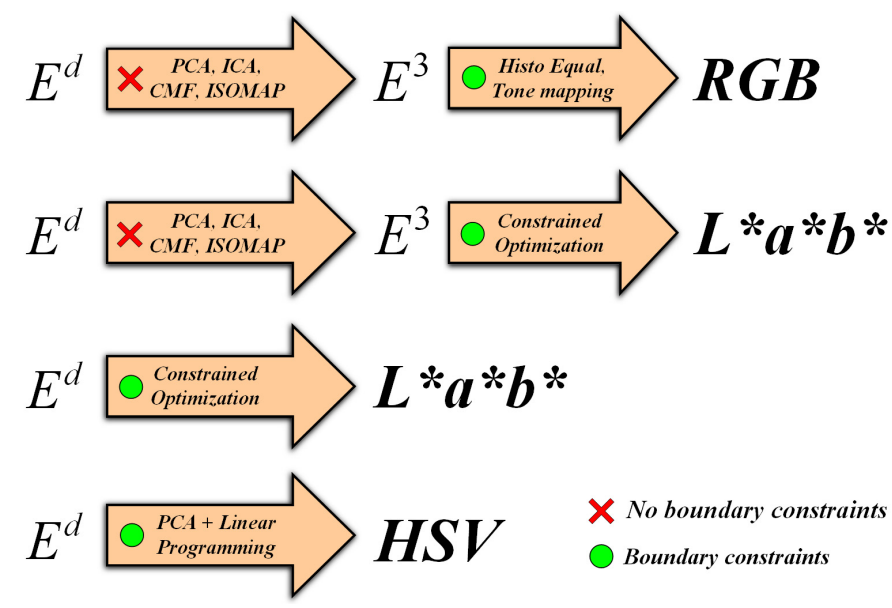

Fig. 2. Four different strategies to address the hyperspectral image visualization problem. The state of the art is shown in the top row. Two possible alternatives are shown in row 2 and 3. Our approach is shown in row 4 (These alternatives are discussed in section III-B).
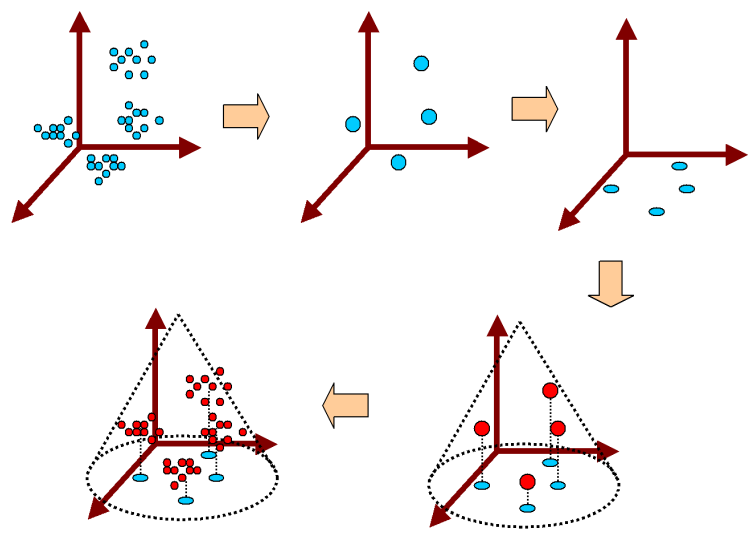

Fig. 3. Pipeline of our framework. Step 1: The points in high dimension are first clustered and representatives for clusters are extracted; Step 2: Representatives are projected to 2d; Step 3: the coordinates in the third dimension are computed using linear programming; Step 4: Interpolation of other points.

and parameter settings for number of clusters $M$ are evaluated in section V.

2) Dimension Reduction: In this stage we want to map the representative points to the HSV color space. Our solution is a two-step algorithm that is tradeoff between computation speed and quality according to the metrics $\gamma$ and $\delta$. First, we project the representative points onto a 2D plane using principle component analysis. The points are then enclosed by a circle which constitutes the boundary in the hue-saturation plane of the HSV color space. Second, we employ a convex optimization to assign intensity values to the representative points.

3) Interpolation and Color Mapping: The hue-saturation components of the remaining points are computed by projecting to the $2 \mathrm{D}$ plane used in the previous step. The intensity component of a point is decided by the distance to its representative point. Finally, all points are mapped into the HSV cone.
4) Interactive Visualization: We provide a suite of interactive tools to explore and analyze a hyperspectral dataset. We provide three types of tools: 1) linear transformations of the color space. 2) spatial lenses so that the user can interactively select a subregion of the image and recalculate a mapping that enhances the visual discriminability of the features in the subregion. 3) Spectral lenses that enhance the visual discriminability of pixels similar to a user specified spectral signature.

\section{Methodology}

\section{A. Preprocessing}

The input to the preprocessing step is the original data set, a high dimensional tensor $T_{d} \in R^{w \times h \times d}$ where each of the $w \times h$ pixels is described by a vector $X_{i}$ with $d$ spectral samples. The output of the preprocessing step is the following clustering information: 1) a $w \times h$ integer map with values ranging from 1 to $M$. The integer is the cluster id for the corresponding pixel, 2) the centroid of each cluster, denoted as $\left\{R_{1}^{d}, R_{2}^{d}, \ldots R_{M}^{d}\right\}$, and 3) the average Euclidean distance of all points to their representative point, denoted as $\left\{r_{1}, r_{2}, \ldots r_{M}\right\}$.

We implemented dimension reduction as an option to accelerate the clustering. We use PCA to first reduce the number of bands and keep $99.9 \%$ of information from the original data sets. This results in keeping the first 10 to 20 eigenvectors. The clustering algorithm is conducted in the projected subspace. This acceleration is fairly conservative and has negligible influence on the output. Therefore, we use it for all results in this paper. We use a $\mathrm{C}$ implementation of the k-means and the median cut clustering algorithm. For both algorithms we tried various settings for number of clusters $(M)$. We found that choosing median cut clustering with $M=50$ gives a good balance between speed and quality of the mapping. We analyze clustering performance and the influence of parameter settings in section $\mathrm{V}-\mathrm{C}$.

\section{B. Dimension Reduction}

1) Problem Formulation: Our method for dimension reduction starts from a multidimensional scaling perspective. Given the spectral samples in original $d$-dimensional Euclidean space denoted as $\left\{X_{1}, X_{2}, \ldots X_{n}\right\}$, each of them being a vector in ddimensions, we try to map them to three-dimensional color vectors denoted as $\left\{C_{1}, C_{2}, \ldots C_{n}\right\}$. To introduce the problem, we only consider the goal of preservation of distances and ignore the boundaries of the color space, so that we allow any color vector in unbounded three-dimensional Euclidian space. That means, we want to find a mapping that minimizes the objective function $\mathrm{E}$ :

$$
E=\sum_{i=1}^{N} \sum_{j=i+1}^{N}\left(D_{d}(i, j)-D_{3}(i, j)\right)^{2}
$$

or in compact form:

$$
E=\frac{1}{2}\left\|D_{d}-D_{3}\right\|_{F}
$$

$\mathrm{F}$ denotes the Frobenius norm, $D_{d}$ and $D_{3}$ are matrices so that $D_{d}(i, j)$ denotes the Euclidean distance between $X_{i}$ 
and $X_{j}$ and $D_{3}(i, j)$ denotes the Euclidean distance between $C_{i}$ and $C_{j}$. It is possible to solve this optimization problem using majorization [37] techniques. However, a more popular approach is to center matrix $D_{d}$ and $D_{3}$ first. The centering operator $\tau$ can be computed by $\tau(D)=-H S H / 2$, where $S=D^{2}$ and $H=I-1 / N * O$ with $O$ being a matrix of all ones and $N$ being the number of rows of square matrix $D$. This transforms the problem to minimizing the objective function E:

$$
E=\left\|\tau\left(D_{d}\right)-\tau\left(D_{3}\right)\right\|_{F}
$$

The importance of the centering operator is that it transforms the problem from a non-linear optimization to an eigendecomposition problem. The main idea is to replace distances with dot products. The global optimum of equation 5 is selected as the 3 eigenvectors of matrix $\tau\left(D_{d}\right)$ that are associated with the largest 3 positive eigenvalues. Each color vector $C_{i}$ can map the first three eigenvectors to any combination of red, green, and blue. This approach is called the classical dimensional scaling. It can be shown that the result is exactly the same as performing PCA on the dataset and computing the projection on the 3 principle components associated with the first 3 largest eigenvalues [37]. In summary, PCA is one of the best and most popular $3 \mathrm{~d}$ projections in the sense of classical multidimensional scaling. However, we need to find a mapping that constrains the vectors $C_{i}$ to lie within the boundaries of a color space.

2) Dimension Reduction Using Convex Optimization: The main motivation and technical contribution of our approach is to derive a convex optimization for the dimension reduction step. Our main ingredients are the use of the HSV color space and a novel solution to split the dimension reduction in two steps. The envelop of the HSV color space is a right circular cone whose height equals the radius of the base plane. The HSV space is shown in figure 4. Our algorithm to map the points from the original d-dimensional space into a $3 \mathrm{~d}$ cone shape has the following steps: 1) We first compute the huesaturation component of the representative points using linear projection. 2) Second, we assign the intensity component of the representative points. For the second step we will explain how we model the intensity assignment as a linear programming problem.

3) Projection to $2 D$ hue-saturation space: The goal of this step is to project the representative points $\left\{R_{1}^{d}, R_{2}^{d}, \ldots R_{M}^{d}\right\}$ in the original d-dimensional space to a 2-dimensional space. The projected points are denoted by $\left\{R_{1}^{2}, R_{2}^{2}, \ldots R_{M}^{2}\right\}$. Note that the superscripts denote the dimensionality of the space the points reside in. The $2 \mathrm{~d}$ space will be parallel to the base plane of the HSV color cone. We use a fast projection using the principal component analysis for this step. Second, we need to find a circle in the plane that describes the boundary of the HSV cone. We use the centroid of the points $\left\{R_{1}^{2}, R_{2}^{2}, \ldots R_{M}^{2}\right\}$ as middle point of the circle and set radius $=$ ratio $*$ Far, where Far denotes the distance of the centroid to the farthest point (see figure 5 left). We need to slightly enlarge the circle because we only use representative points at this step and other points will still be further away. We use ratio $=1.2$
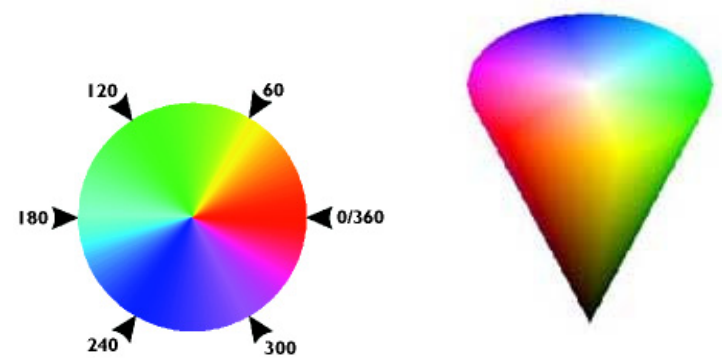

Fig. 4. The HSV color space is bounded by a circular cone. On the left we show a cross section of the cone. Hue ranges from 0 to 360 and describes the spectrum of pure colors, Saturation is the distance to the center of the circle and describes color strength. Adding more white will make the color weaker. On the right we show the cone in 3D. The Value coordinate describes how bright or dark the color is

as an initial heuristic, but the user can modify this parameter interactively. Please note that we do not want to enclose all projected points in the base circle, because this would make our method sensitive to outliers. Up to now the hue-saturation component of the representative points are decided up to a rotation factor. This degree of freedom can also be set by the user during interactive exploration (see section. IV-D.1).

4) Computing the intensity component: In this step we compute the intensity components for all representative points denoted as $\left\{I_{1}, I_{2}, \ldots I_{M}\right\}$. Note that $\left(R_{1}^{2}, I_{1}\right),\left(R_{2}^{2}, I_{2}\right), \ldots\left(R_{M}^{2}, I_{M}\right)$ together fully describe the final projection in $3 \mathrm{~d}$ space $\left(R_{1}^{3}\right),\left(R_{2}^{3}\right), \ldots\left(R_{M}^{3}\right)$. Please note that we use negative intensity values between 0 and -1 since the cone is upside down $(-1$ maps to black). The principal goal is to preserve the mutual distances of representative points in original space as much as possible (see equation 3) while respecting several constraints. The problem can be modeled as a linear programming problem. We will describe our solution in five parts. 1) First we describe the objective function, 2) We show how the objective function can be transformed to map to a convex optimization algorithm, 3) We explain how to incorporate the boundaries of the HSV cone as constraints, 4) We explain how to add cluster separability as constraint, and 5) we show how to solve the problem numerically.

Objective function: The objective in equation 3 is to keep $D_{3}$ as close to $D_{n}$ as possible. At this step in the algorithm we already have a $2 \mathrm{~d}$ projection as a partial solution and we only need to solve for $1 \mathrm{~d}$ coordinates in the third dimension. We denote the pairwise distance matrix for $\left\{R_{1}^{2}, R_{2}^{2}, \ldots R_{M}^{2}\right\}$ in $2 \mathrm{~d}$ space as $D_{2}$, and the pairwise distance matrix for $\left\{I_{1}, I_{2}, \ldots I_{M}\right\}$ as $D_{1}$. In the ideal case $D_{1}(i, j)=\sqrt{\left(D_{d}(i, j)\right)^{2}-\left(D_{2}(i, j)\right)^{2}}$ according to the Pythagorean theorem. Therefore, we want to minimize the following objective function $E$ :

$$
E=\sum_{i=1}^{M} \sum_{j=i+1}^{M}\left|D_{1}(i, j)-\sqrt{\left(D_{d}(i, j)\right)^{2}-\left(D_{2}(i, j)\right)^{2}}\right|
$$

Transforming to a linear programming problem: Even though the term under the square root in equation 6 is a constant, the resulting optimization is still non-convex. The reason for this is the absolute value in $D_{1}(i, j)=\left|I_{i}-I_{j}\right|$. 
Therefore, we cannot solve the minimization problem using linear programming. The key insight to bypass this problem is to decide the order of $\left\{I_{1}, I_{2}, \ldots I_{M}\right\}$ using a reasonable criterion beforehand. This order becomes the first set of constraints:

$$
\text { if } \quad \operatorname{Dist}(i) \geq \operatorname{Dist}(j) ; \quad I_{i} \geq I_{j}
$$

We will discuss the meaning of $\operatorname{Dist}(i)$ and why we impose these constraints shortly. Now $D_{1}(i, j)=I_{i}-I_{j}$ when $I_{i} \geq I_{j}$, or $D_{1}(i, j)=I_{j}-I_{i}$ when $I_{i}<I_{j}$. The absolute value disappears and the problem is reduced to a linear programming problem.

Cone boundary Constraints: The second set of constraints are straightforward: we want to restrict the position of the points in final $3 \mathrm{~d}$ space within a cone shape. Let's denote $\operatorname{Dist}(i)$ as the distance from point $R_{i}^{2}$ to the origin of the base circle, since the height of the cone is equal to the radius of base circle, we know that $\left|I_{i}\right|+\operatorname{Dist}(i) \leq$ Radius must be satisfied, shown in figure 5 right. Therefore, if Range $(i)=$ Radius $\operatorname{Dist}(i)$, the following two constraints must be applied to make $R_{i}^{3}$ stay in a cone:

$$
I_{i} \leq 0 \quad \text { and } \quad I_{i} \geq- \text { Range }(i)
$$

Transforming to LP problem constraints: Now we look back at the first set of constraints and explain why they make sense. Since the smaller the $\operatorname{Dist}(i)$, the larger Range $(i)$ is, we intentionally make the final $3 \mathrm{~d}$ points appear more like a cone shape by arranging their intensity value in descending order according to $\operatorname{Dist}(i)$. It is worth mentioning that other methods exist to decide the order of $\left\{I_{1}, I_{2}, \ldots I_{M}\right\}$. For example, we can use the total energy in the original space: $\left|R_{i}^{d}\right|_{1}$ to order them. We tested this setting and final results do not form a good cone shape.

Cluster separability constraints: The third and last set of constraints is designed to separate important points. Remember the we define $r_{i}$ as the average distance to the $i^{t h}$ representative point for all points belonging to that cluster. We define two representative points to be well separated if $r_{i}+r_{j} \leq D_{d}(i, j)$. We want the cluster center to remain well separated in the final 3d space: $r_{i}+r_{j} \leq D_{3}(i, j)$. This boils down to the following constraint:

$$
\begin{array}{r}
\text { if } \quad r_{i}+r_{j} \leq D_{d}(i, j) \quad \text { then } \\
\sqrt{\left(r_{i}+r_{j}\right)^{2}-\left(D_{2}(i, j)\right)^{2}} \leq D_{1}(i, j)
\end{array}
$$

Solve convex optimization: Now we have fully set up the linear programming model: minimize eq.(6) subject to eq.(7), eq.(8) and eq.(9). We solve it with CLP $^{1}$. CLP is a C++ library of several linear programming solvers. We use the Primal-Dual method implemented in the library. This is not the fastest method but it works fast enough for our application. Alternatively, instead of minimizing eq.(6), we can minimize the following term $\bar{E}$ :

\footnotetext{
${ }^{1}$ https://projects.coin-or.org/Clp
}

$$
\bar{E}=\max _{i=1 \ldots M, j=i+1, \ldots M}\left|D_{1}(i, j)-\sqrt{\left(D_{d}(i, j)\right)^{2}-\left(D_{2}(i, j)\right)^{2}}\right|
$$

This formulation uses the infinity norm instead of the 1-norm. We believe it gives a better theoretical guarantee. Therefore, we use this objective function for all results in this paper. The derivation of the corresponding linear programming problem is analogous to the derivation presented in this section.
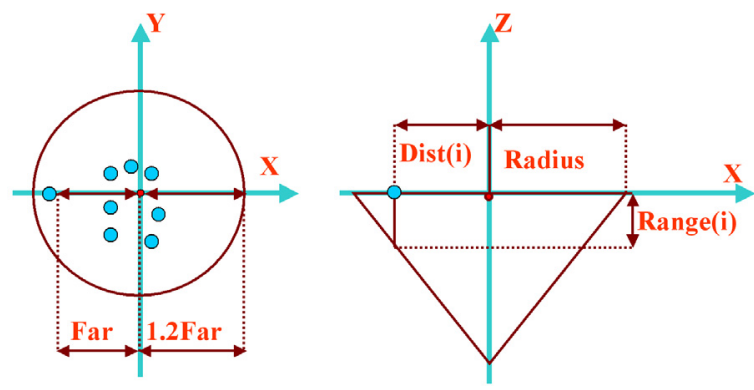

Fig. 5. Left: The $2 \mathrm{~d}$ projection in enclosed in a circle; Right: the range of the intensity for each point. The $\mathrm{X}-\mathrm{Y}$ plane is the hue-saturation plane and the $\mathrm{Z}$ axis corresponds to value from the HSV color space.

\section{Interpolation and Color Mapping}

1) Interpolate All Points: At this stage, the coordinates of the representative points are fully determined in $3 \mathrm{~d}$ space (HSV color space). In this step we will map all the remaining points based on the location of the representative points. The coordinates in the hue-saturation plane are decided by projecting all other points in original space to the same plane using the first two principle components we have used for the representative points. Note that alternatively we can use the first two principle components of all data points with similar computation time. However, performing PCA on all points will bias favorably towards the separability of clusters with a larger number of members. The intensity $I_{p}$ for a particular pixel $P^{d}$ is decided by the following formula:

$$
I_{p}=I_{i}+f \operatorname{lag} * \sqrt{E \operatorname{dist}\left(P^{d}, R_{i}^{d}\right)-\operatorname{Edist}\left(P^{2}, R_{i}^{2}\right)}
$$

In the formula $i$ denotes which cluster $P^{d}$ belongs to. $I_{i}$ is the intensity value we have decided in the previous section. $E \operatorname{dist}(p, i)$ denotes the Euclidean distance between $p$ and $i$ in original space. flag is either 1 or -1 denoting whether pixel $I_{p}$ should be darker or lighter than its representative points intensity $I_{i}$. flag is decided by comparing the sum of all bands values for this pixel to the sum of all bands for the representative point in the original space. In figure 6 we show the mapped cone shape of a real data set (LunarLake02).

2) Fit the Color Cone to Data Cone: Now the basic idea is to make the color cone big enough to enclose the data cone such that all points are mapped to different colors. This corresponds to the largest red cone shown in figure 7 left. To fit the cone so that it encloses all data points might not be the best strategy due to outliers in the data. The display strategy 


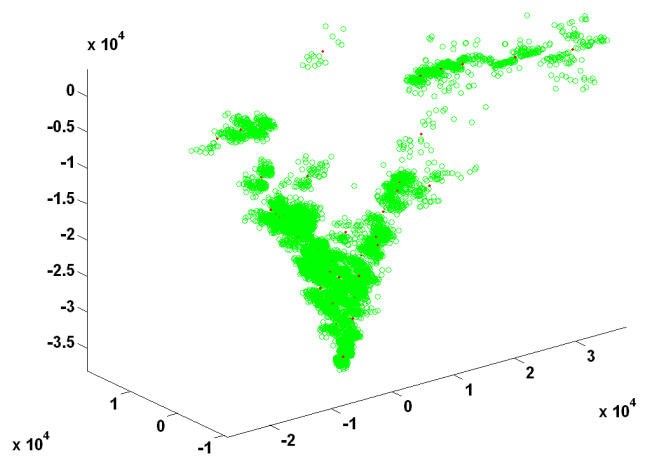

Fig. 6. All data points are mapped to 3D Euclidean space using our method described in section IV. Note how they form a cone shape. The units are Euclidean distances in the original dataset.

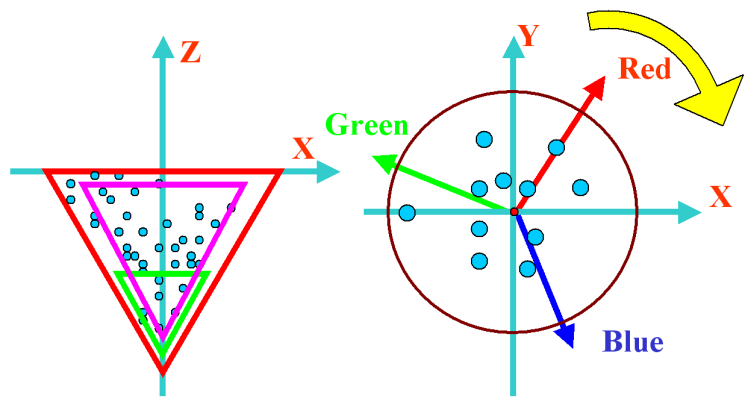

Fig. 7. Left: how to fit the color cone to the data cone; Right: The HSV cone can be rotated by the user.

is to allow the user to shift the color cone up and down along the $\mathrm{Z}$ axis and scale the radius of the cone to enhance different parts of the data. This idea is illustrated using the green and pink cone in figure 7 left. Data points outside the color cone will be clamped to the boundary of the cone.

\section{Interactive Visualization}

In the following we outline three tools useful for interactive visualization: 1) interactive tone rotation, 2) spatial lenses, and 3) spectral lenses.

1) Interactive Tone Rotation: The user can rotate the orientation of the base plane. This is shown in figure 7 right. Note that the intensities do not need to be re-calculated due to the symmetric structure of the cone. Remapping can be computed in less than a second for millions of points. We found that functionality very helpful to enhance the contrast and to change the visualization to more aesthetic color choices.

2) Spatial Lens: We propose to use spatial lenses to improve the contrast in selected regions. We can specify a region interactively and recompute a mapping only on that part of the data. The region is treated as an input image itself. Although we still use the same thematic labeling which we got from the preprocessing step for the whole data set, we re-map these colors to a cone shape in itself. This allows us to enhance smaller features while locally keeping proportional perceptual distances. A local mapping is also achievable with PCA on the subregion. The PCA will also perform well if the selected region is sufficiently small. This is illustrated in figure 8:
We see in CMF, the features are distinguished fairly well, however, the overall contrast can be improved. In the PCA without enhancement, the features are not easily distinguished, especially for the slim white branches at the upper right corner. In the PCA with enhancement setting, the results are very colorful and features are distinguishable. However, the color mapping does not correspond to the true distance and is misleading. For example, at the middle part near the bottom we see a big contrast of yellow and pink areas, even though these regions have similar spectral signatures. More results for the spatial lens are available in section $\mathrm{V}$.

3) Spectral Lens: Another tool we offer is to enable the user to interactively pick one particular pixel in the result image and highlight pixels that have a similar spectral signature to the pixel in original space. This way we can use a larger portion of the color space for selected features and their surrounding. We implement this functionality by creating a new cluster whose centroid is set to be the pixel the user has picked and the members to be all the pixels within some radius in original space. We call this new center $R_{M+1}^{d}$. Note that the thematic labeling map is changed due to insertion of the new cluster. Then the mapping algorithm is re-computed with a new constraint specifying $I_{M+1} \geq I_{i}$ for all $i \leq M$. The goal of this constraint is to guarantee that the neighborhood of this pixel is "highlighted". The results are shown in the middle image of the top row of figure 11 and figure 12. Note that there are also some other algorithms available to achieve a similar goal. For example, a well known technique is to set the hue and saturation components of all the points within a distance directly to the same as that of the selected pixels and keep their intensity values untouched. We argue that our approach is more systematic by intentionally making the rest of the points less "highlighted". Additionally, our approach can provide more hue-saturation variations within the cluster.

\section{Results}

\section{A. Implementation Details}

We implemented our algorithm in Matlab on a 3.6Ghz Xeon processor. The algorithm takes less than 1000 lines of code including the display, input, and output routines. While Matlab greatly helps the simplicity of implementation, a complete $\mathrm{C}++$ implementation would be faster. However, since our algorithm speed is already very competitive, we opted against further low level optimizations and porting our code to $\mathrm{C}++$.

We use the AVIRIS data which is available for download online. ${ }^{2}$ Each dataset has 219 bands ranging from 400 to 2500 $\mathrm{nm}$ with uniform steps of $10 \mathrm{~nm}$. The image size is usually around 500 by 500 . We picked seven data sets: the scene 01 , 02 and 03 from site Moffett Field, scene 01 and 02 from site Lunar Lake and scene 01 and 02 from site Cuprite.

We compared our method against four previously published algorithms: color matching functions $(\mathrm{CMF})$, principal component analysis (PCA), principal component analysis with outlier reduction (PCA 2\%), principal component analysis with histogram equilization (PCA HE), spectral band sampling

\footnotetext{
${ }^{2}$ http://aviris.jpl.nasa.gov/html/aviris.freedata.html
} 

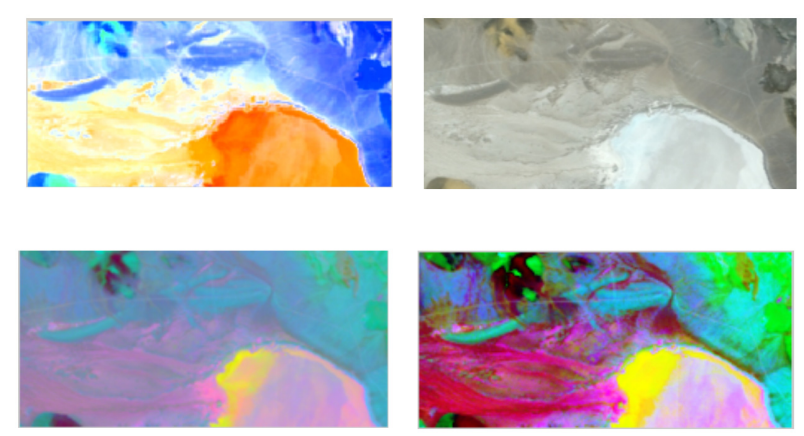

Fig. 8. Top left: result using our spatial lens; Top right: result of CMF, zoomed in; Bottom left: result using PCA; Bottom right: result using PCA with histogram equalization The rectangular region is selected within the yellow border in the top left image of figure 12. Algorithmic details are described in section V-A.

(SBS), and ISOMAP. Note that ISOMAP is a nonlinear dimension reduction method. ISOMAP preserves geodesic distances rather than Euclidean distances. Therefore, we only visually compare ISOMAP results to ours.

CMF linearly projects the dataset using three fixed basis vectors which are called color matching functions in [7]. The PCA method uses the largest three principle components $(P 1, P 2, P 3)$ to map all pixels to three-dimensional Euclidian space and then linearly scales the whole dataset so that it fits into the (unit) RGB cube. The projection on $(P 1, P 2, P 3)$ is mapped to $(R, G, B)$ channels respectively. The PCA $2 \%$ method also uses PCA and scaling to the RGB cube, but instead of linearly scaling, 2 percent of the pixels at the ends of each channel are saturated in order to enhance the contrast. A saturated pixel value is one that is moved outside the RGB cube through scaling and subsequently clamped to the boundaries of the cube. Similarly, PCA with histogram equalization uses PCA for dimension reduction and scaling to the RGB cube, but then uses histogram equalization for each color channel. SBS is the simplest algorithm that directly maps the 6th, 20th and 40th bands to RGB color bands respectively. Finally, we also implemented a method that uses ISOMAP to do dimension reduction. The implementation of ISOMAP follows [38]. The algorithm takes half an hour to get a mapping for an image of $500 \times 500 \times 219$. The implementation of ISOMAP in [28] reports 4.4 hours on images with $1.8 \times 10^{6}$ samples where each sample has 124 bands. Since the algorithm complexity is $O\left(N \log ^{2}(N)\right)$, we estimate the running time on an image of $500 \times 500 \times 124$ will take at least 500 seconds. We therefore believe that current implementations do not meet the interactive display requirement.

\section{B. Quantitative Comparison}

We measure the quality of the mapping based on the two metrics $\gamma$, indicating the preservation of distances (see equation 1) and $\delta$ indicating the separability of features (see equation 2). The result for $\gamma$ is shown in table I and result for $\delta$ is shown in table II. Note that a good mapping should have $\gamma$ close to 1 and $\delta$ as high as possible. To accelerate the quantitative comparison we randomly subsample pixels so that in each row and column only every fifth pixel is used in the
TABLE I

COMPARISON OF CORRELATION $\gamma$

\begin{tabular}{|c|c|c|c|c|c|}
\hline & Our & CMF & PCA & PCA2\% & PCA HE \\
\hline Moffett01 & 0.96 & 0.94 & 0.91 & 0.68 & 0.46 \\
\hline Moffett02 & 0.96 & 0.79 & 0.92 & 0.51 & 0.35 \\
\hline Moffett03 & 0.93 & 0.69 & 0.96 & 0.68 & 0.41 \\
\hline LunarLake01 & 0.95 & 0.82 & 0.92 & 0.53 & 0.21 \\
\hline LunarLake02 & 0.84 & 0.81 & 0.95 & 0.37 & 0.27 \\
\hline Cuprite01 & 0.90 & 0.87 & 0.91 & 0.55 & 0.32 \\
\hline Cuprite02 & 0.91 & 0.88 & 0.95 & 0.43 & 0.28 \\
\hline
\end{tabular}

TABLE II

Comparison of Average Distance $\delta$

\begin{tabular}{|c|c|c|c|c|c|}
\hline & Our & CMF & PCA & PCA2\% & PCA HE \\
\hline Moffett01 & 38.2 & 16.4 & 13.0 & 50.3 & 81.4 \\
\hline Moffett02 & 53.9 & 30.6 & 12.4 & 48.5 & 77.2 \\
\hline Moffett03 & 25.5 & 30.5 & 9.7 & 44.7 & 75.9 \\
\hline LunarLake01 & 50.9 & 5.6 & 10.9 & 52.5 & 85.3 \\
\hline LunarLake02 & 59.8 & 7.3 & 15.6 & 43.8 & 80.1 \\
\hline Cuprite01 & 52.1 & 4.8 & 10.9 & 48.4 & 80.7 \\
\hline Cuprite02 & 73.0 & 7.7 & 13.9 & 53.2 & 81.5 \\
\hline
\end{tabular}

computation of the pairwise distances. That means only $4 \%$ of the pixels are used.

The values of SBS are not competitive and we did not include them in the table. Since ISOMAP preserves geodesic distances rather than Euclidean distances it is not meaningful to apply the correlation metric to ISOMAP results.

The comparison of $\gamma$ values reveals that PCA 2\% and PCA HE strongly exaggerate features and therefore have a low correlation score. CMF produces solid results, but our method and PCA are generally better than CMF. Even though our method is better than PCA in some cases, we consider PCA to be the most stable according to $\gamma$ and therefore the best method to preserve the distances. We consider our method the second best.

However, the comparison of $\delta$ shows the significant drawback of PCA. The separability of features is low and this results in dark images that are not useful for visualization. The $\delta$ values are a factor of $3-5$ times lower than our method. The $\delta$ values of CMF are comparable to our method for the first three datasets, but other datasets exhibit $\delta$ values that are up to ten times lower. As expected, PCA $2 \%$ is comparable to our method and PCE HE has the best $\delta$ values. However, note that these two algorithms did not perform well according to our metric $\gamma$ and are generally not distance preserving.

We conclude that our method is the best trade-off and achieves both goals of preserving spectral distances and separating features in the visualization.

\section{Parameter Selection for Our Algorithm}

We evaluated the parameter for the number of clusters $M$ and the two implemented clustering algorithms k-means and median cut. We use two data sets Moffett01 and LunarLake01 for the evaluation and for each of the two data sets we additionally create a $25 \%$ and $50 \%$ downsampled version (in spatial dimensions only) giving a total of 6 data sets. The original size of both the datasets is $512 \times 614 \times 219$. 
TABLE III

COMPARING RUNNING TIME IN SECONDS OF DIFFERENT NUMBER OF CLUSTERS AND CLUSTERING METHODS FOR OUR METHOD (UNITS ARE IN SECONDS)

\begin{tabular}{|c|l|l|l|l|l|l|l|}
\hline & \multicolumn{3}{|c|}{ K-means } & \multicolumn{3}{c|}{ Median cut } \\
\hline Number of clusters (M): & 25 & 50 & 100 & 25 & 50 & 100 \\
\hline \multirow{3}{*}{ Mof01 25\% } & Cluster & 2.9 & 7.2 & 11.8 & 0.3 & 0.2 & 0.2 \\
\cline { 2 - 8 } & Optimize & 0.4 & 5.7 & 82.6 & 0.4 & 6.3 & 70 \\
\cline { 2 - 8 } & Total & 3.3 & 12.9 & 94 & 0.7 & 6.5 & 70 \\
\hline \multirow{3}{*}{ Mof01 50\% } & Cluster & 17.4 & 47.8 & 86.6 & 0.9 & 1.0 & 1.1 \\
\cline { 2 - 8 } & Optimize & 0.4 & 5.9 & 84 & 0.4 & 7.2 & 77 \\
\cline { 2 - 8 } & Total & 17.8 & 54 & 171 & 1.3 & 8.2 & 78 \\
\hline \multirow{3}{*}{ Mof01 100\% } & Cluster & 115 & 427 & 917 & 5.2 & 4.8 & 5.4 \\
\cline { 2 - 8 } & Optimize & 0.4 & 6 & 87 & 0.6 & 5.9 & 66 \\
\cline { 2 - 8 } & Total & 115 & 433 & 1000 & 5.8 & 10.7 & 71.4 \\
\hline \multirow{3}{*}{ Lak01 25\% } & Cluster & 3.6 & 8.2 & 14.2 & 0.2 & 0.2 & 0.2 \\
\cline { 2 - 8 } & Optimize & 0.5 & 5.8 & 79 & 0.5 & 6.3 & 92 \\
\cline { 2 - 8 } & Total & 4.1 & 14 & 94 & 0.7 & 6.5 & 92 \\
\hline & Cluster & 23.4 & 59.2 & 125 & 1.0 & 0.9 & 0.9 \\
\cline { 2 - 8 } & Optimize & 0.4 & 5.6 & 79.9 & 0.6 & 7.9 & 75.6 \\
\cline { 2 - 8 } & Total & 23.8 & 60 & 205 & 1.6 & 8.8 & 77 \\
\hline & Cluster & 126 & 523 & 1037 & 4.6 & 4.8 & 4.5 \\
\cline { 2 - 8 } & Optimize & 0.4 & 6.2 & 86.8 & 0.5 & 7.4 & 89.6 \\
\cline { 2 - 8 } & Total & 126 & 529 & 1124 & 5.1 & 12.2 & 94 \\
\hline
\end{tabular}

TABLE IV

COMPARING CORRELATION $\gamma$ AND AVERAGE DISTANCE $\delta$ OF DIFFERENT NUMBER OF CLUSTERS AND CLUSTERING METHODS FOR OUR METHOD

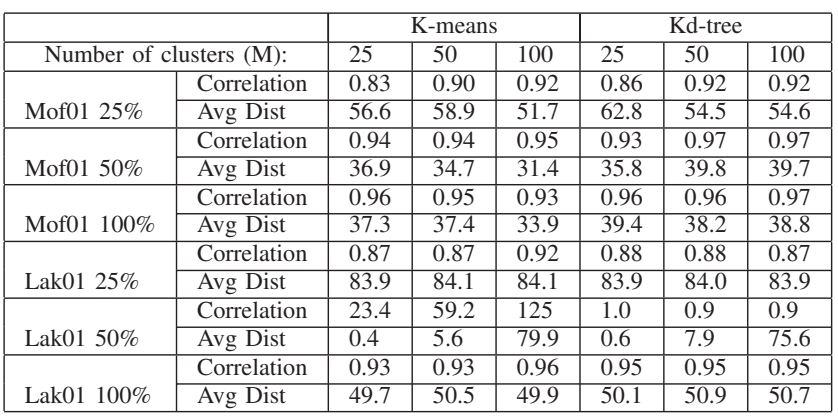

In table III we compare the running time of different settings for number of clusters, clustering algorithm, and input image size. The two most time consuming steps in our algorithm are clustering and convex optimization on representative points (Interactive visualization tools, such as the rotation of the color wheel and the spatial lens on a small subset of the data have response times of less than 0.1 seconds). The table shows that k-means clustering is much slower than median cut. Note that our recommended setting of $M=50$ results in visualization times of about 10 seconds which is reasonable for interactive software. We also see that increasing the image size has less impact on the running time than increasing the number of clusters.

In table IV we present a quantitative evaluation of the parameter setting. We can observe that the quality for using 50 clusters is only slightly worse than using 100 clusters.

\section{Visual Comparison}

In figure 9 and figure 10, we show the comparison between our algorithm and the CMF algorithm. While CMF tries to preserve the influence of the visible spectral bands we can see that several features are lost in the visualization.
In figure 11 we see the results of different algorithms on dataset Moffettfiled02. In the top left image, which is our result, the urban area looks very clear, with several details non-observable in other methods. The lake is relatively dark, but can be made clearer with a spatial or spectral lens. Please also note how the PCA without any enhancement produces a visualization that is too dark as indicated by low values of $\delta$ in our quantitative comparison. In figure 12 we show a visual comparison of selected algorithms on the Lunarlake02 data set. see that in our result, features are again easily distinguishable. For this data set we use PCA with histogram equalization (PCA HE). Note that histogram equalization can provide colorful results but that the interpretation is difficult because the distances in original spectral space are not preserved resulting in low values of $\gamma$.

In figure 13 we see three more examples of the application of a spatial lens. For each example we compare our method to CMF, PCA, and PCA HE. A few remarks about the results. Example one: the curved strip in the left middle part of the image is very clear, while CMF fails to show this. The straight strip does not show up in the PCA without enhancement. In PCA with enhancement, the body of two parts in the lake has too much perceptual difference: one part is pink and the other part is green. From the original data we know they should not be that dissimilar to each other. Example two: both our method and CMF appear to do a good job, but in PCA without enhancement the features are less clear. In PCA HE, the two different kinds of materials are mapped to blue and red respectively, which is not desirable. In example three $\mathrm{CMF}$ cannot distinguish the features very well, while our method and PCA without enhancement get similar result.

\section{DISCUSSION}

Advantages: This new framework provides a good visualization result for hyperspectral images while avoiding distortion of significant features. It also provides real-time interaction to further facilitate exploration. Based on the visual and quantitative comparison we argue that we outperform the state-of-the-art techniques.

Limitations and Future Work: There are several aspects of our algorithm that we want to improve in future work. One limitation of the current algorithm is that it does not try to map high dimensional pixel signatures to natural colors. Although we can partially meet this requirement by rotating the color wheel to make a particular part of the image look natural, we do not have a systematic way to guarantee that all features satisfy this criterion at the same time. We also would like to experiment with using ICA instead of PCA for projecting the colors to the $2 \mathrm{D}$ plane.

It is also worth mentioning that the two goals we set up at the beginning, namely, preserving spectral distance and obtaining high feature separability may be two contradictory goals. In the current algorithm, these two goals are unified in the optimization process by explicitly setting the preservation of spectral distances as the objective function and casting feature separability as a set of constraints. We would like to explore the possibility to put both goals in the objective 

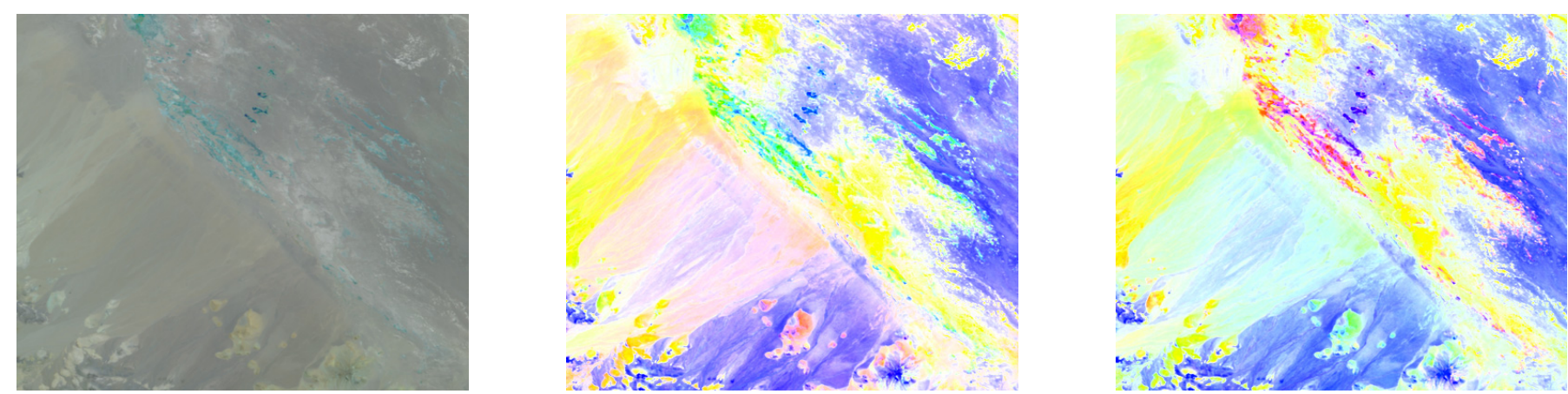

Fig. 9. Left: CMF result, $\gamma=0.82, \delta=5.6$; Middle: Our result with Kd-tree, $\gamma=0.95, \delta=50.9$; Right: Our result with $\mathrm{K}$-means, $\gamma=0.95, \delta=50.5$.
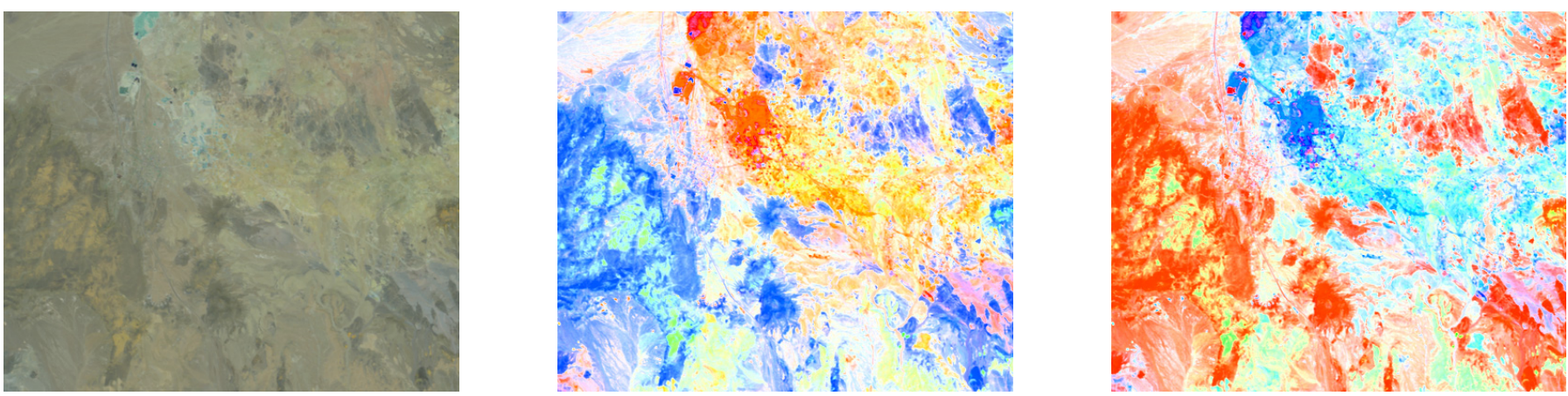

Fig. 10. Left: CMF result, $\gamma=0.88, \delta=7.7$; Middle: Our result with Kd-tree, $\gamma=0.91, \delta=73.0$; Right: Our result with K-means, $\gamma=0.90, \delta=76.2$.
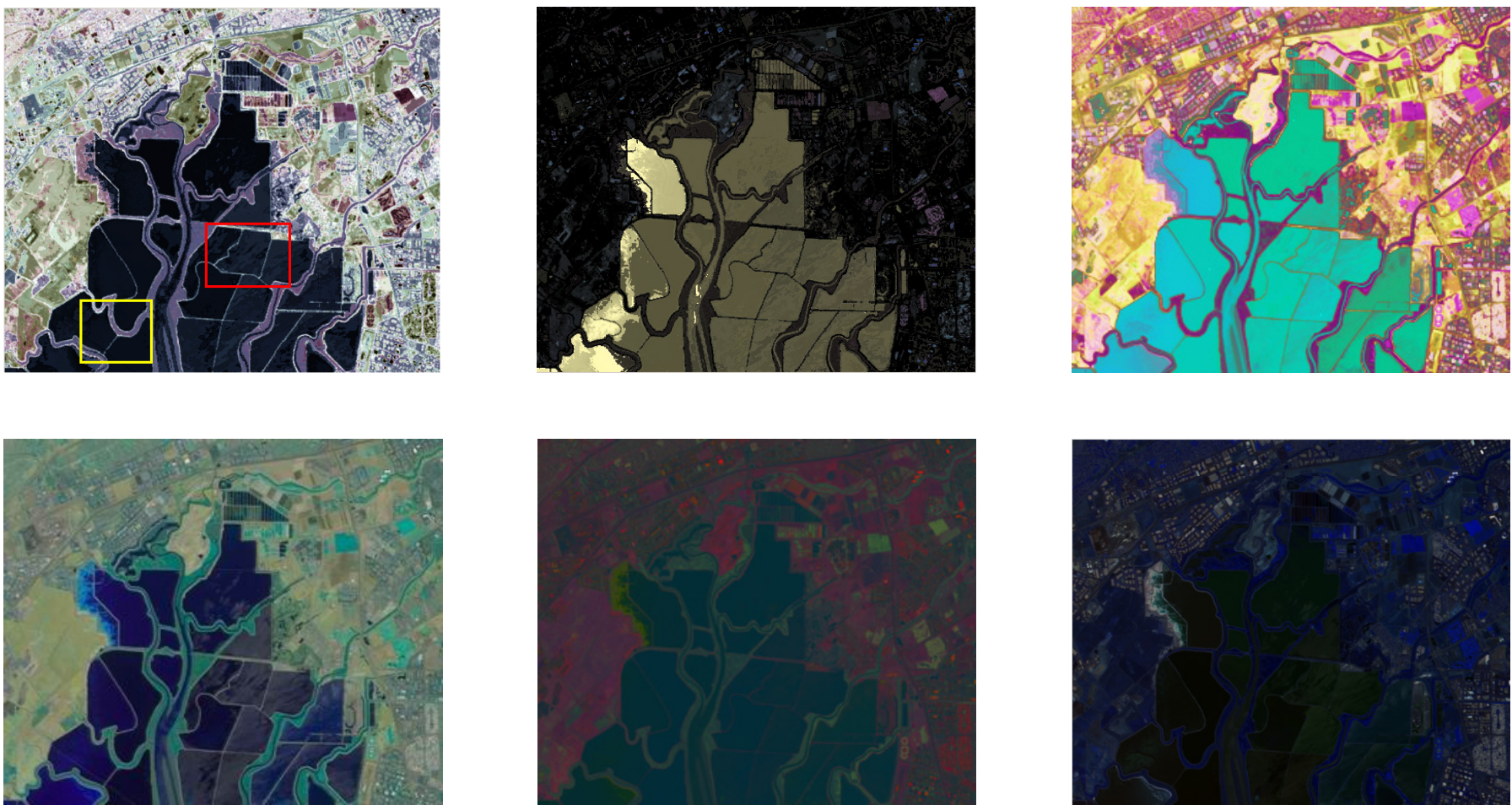

Fig. 11. Top Left: Result using our method; Top Middle: Result using our spectral lens; Top right: Result using ISOMAP; Bottom Left: Stretched Color Matching Functions [7]; Bottom Middle: PCA without enhancement; Bottom Right: Result using 3 bands directly chosen from original data. The data set is scene 2 from Moffettfield.

function with a parameter to balance their relative weights in the future.

\section{CONCLUSION}

In this paper we propose a new framework for hyperspectral image visualization. We are the first to consider the final color space in our computation and therefore we are able to derive 

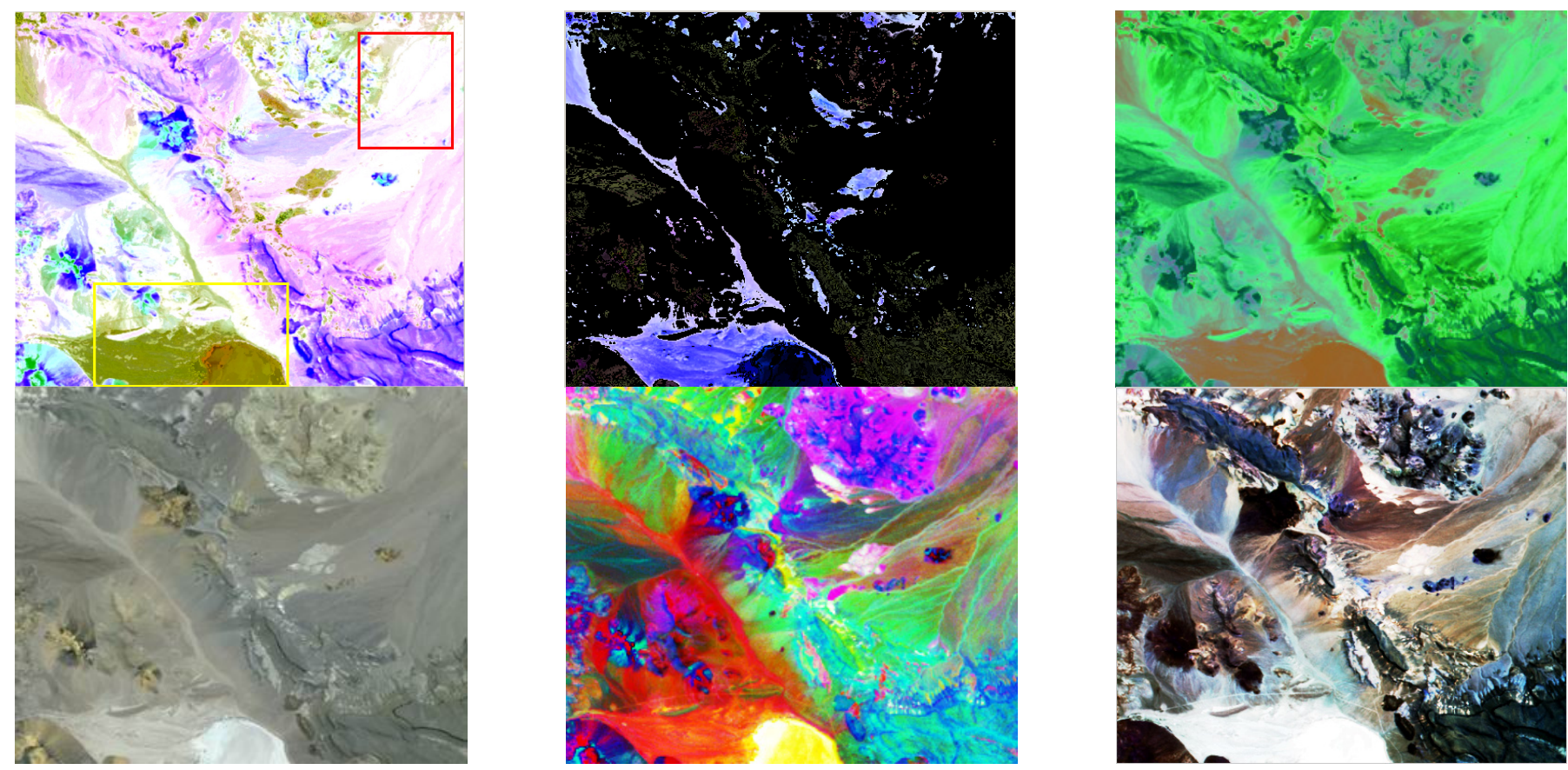

Fig. 12. Top Left: Result using our method; Top Middle: Result using our spectral lens; Top right: Result using ISOMAP; Bottom Left: Stretched Color Matching Functions [7]; Bottom Middle: PCA using histogram equalization; Bottom Right: Result using 3 bands directly chosen from original data. The data set is scene 2 from Lunarlake.
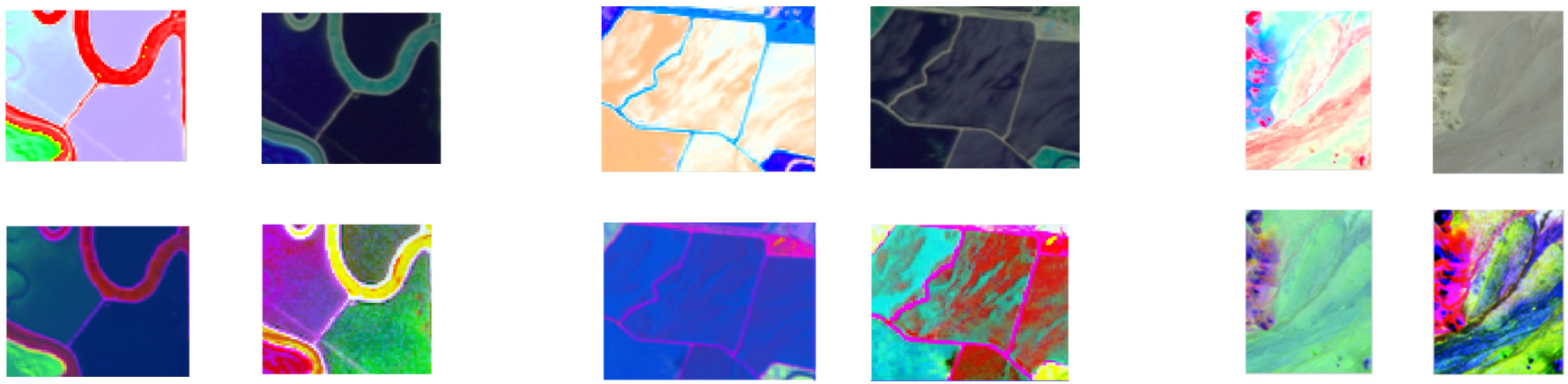

Fig. 13. The figure shows the application of the spatial lens on three examples. Each of the three examples compares four methods: our algorithm in the top left, CMF in the top right, PCA in the bottom left and PCA HE in the bottom right. Left: The spatial lens is applied to the yellow rectangle shown in the left image of figure 11. Middle: red rectangle in top left image of figure 11. Right: red rectangle in the top left image of figure 12. For each example the layout is the same as figure 8 .

a higher quality mapping than previous work. Experiments show the visual quality of the final mapping improves over state-of-the-art approaches. The framework also provides some interaction abilities which are important for a human analyst to explore the data.

\section{ACKNOWLEDGMENTS}

This research was financially supported by NGA HM158208-BAA-0003, NGA HM1582-05-1-2004, and NSF IIS 0612269. The authors would also like to thank Jieping Ye and Stefan Jeschke for helpful discussions.

\section{REFERENCES}

[1] MultiSpec, "http://cobweb.ecn.purdue.edu/ biehl/multispec/description.html."

[2] ENVI, "http://www.ittvis.com/envi/."

[3] Geomatics, "http://www.pcigeomatics.com/."

[4] TnTlite, "http://www.microimages.com/tntlite/."
[5] HyperCube, "http://www.tec.army.mil/hypercube/."

[6] HIAT, "http://www.censsis.neu.edu/software/hyperspectral/hyperspectral.html."

[7] N. Jacobson and M. Gupta, "Design goals and solutions for display of hyperspectral images," IEEE Transactions on Geoscience and Remote Sensing, vol. 43, no. 11, pp. 2684-2692, November 2005.

[8] J. Wang. and C.-I. Chang., "Independent component analysis-based dimensionality reduction with applications in hyperspectral image analysis," IEEE Transactions on Geoscience and Remote Sensing, vol. 44, no. 6, pp. 1586-1600, June 2006.

[9] Y. Zhu, P. K. Varshney, and H. Chen, "Evaluation of ica based fusion of hyperspectral images for color display," Information Fusion, 2007 10th International Conference on, pp. 1-7, 9-12 July 2007.

[10] A. A. Gooch, S. C. Olsen, J. Tumblin, and B. Gooch, "Color2gray: salience-preserving color removal," in SIGGRAPH '05: ACM SIGGRAPH 2005 Papers. New York, NY, USA: ACM Press, 2005, pp. 634-639.

[11] G. R. Rasche, K. and J. Westall, "Re-coloring images for gamuts of lower dimension," Computer Graphics Forum, vol. 24, no. 3, pp. 423432, 2005.

[12] D. A. Forsyth and J. Ponce, Computer Vision: A Modern Approach. Prentice Hall Professional Technical Reference, 2002.

[13] R. Smith, Ed., Analyzing Hyperstectral Images with TNTmips. Mi- 
croimages, 2006.

[14] J. Tyo, A. Konsolakis, D. Diersen, and R. Olsen, "Principal-componentsbased display strategy for spectral imagery," IEEE Transactions on Geoscience and Remote Sensing, vol. 41, no. 3, pp. 708-718, March 2003.

[15] J. Wang and C. Chang, "Independent component analysis-based dimensionality reduction with applications in hyperspectral image analysis," IEEE Transactions on Geoscience and Remote Sensing, vol. 44, no. 6, pp. 1586-1600, June 2006.

[16] Q. Du, N. Raksuntorn, S. Cai, and R. Moorhead, "Color display for hyperspectral imagery," IEEE Transactions on Geoscience and Remote Sensing, vol. 46, no. 6, pp. 1858-1866, June 2008.

[17] S. Cai, Q. Du, and R. Moorhead, "Hyperspectral imagery visualization using double layers," IEEE Transactions on Geoscience and Remote Sensing, vol. 45, no. 10, pp. 3028-3036, October 2007.

[18] K. Rasche, R. Geist, and J. Westall, "Detail preserving reproduction of color images for monochromats and dichromats," IEEE Comput. Graph. Appl., vol. 25, no. 3, pp. 22-30, 2005.

[19] R. Mantiuk, K. Myszkowski, and H.-P. Seidel, "A perceptual framework for contrast processing of high dynamic range images," ACM Trans. Appl. Percept., vol. 3, no. 3, pp. 286-308, 2006.

[20] J. B. Tenenbaum, V. de Silva, and J. C. Langford, "A global geometric framework for nonlinear dimensionality reduction." Science, vol. 290, no. 5500, pp. 2319-2323, December 2000. [Online]. Available: http://dx.doi.org/10.1126/science.290.5500.2319

[21] S. T. Roweis and L. K. Saul, "Nonlinear dimensionality reduction by locally linear embedding," Science, vol. 290, no. 5500, pp. 2323-2326, December 2000. [Online]. Available: http://dx.doi.org/10.1126/science.290.5500.2323

[22] M. Belkin and P. Niyogi, "Laplacian eigenmaps for dimensionality reduction and data representation," Neural Comput., vol. 15, no. 6, pp. 1373-1396, 2003

[23] D. Donoho and C. Grimes, "Hessian eigenmaps: locally linear embedding techniques for high dimensional data," Proc. of National Academy of Sciences, vol. 100, no. 10, pp. 5591-5596, 2003. [Online]. Available: citeseer.ist.psu.edu/donoho03hessian.html

[24] F. Sha and L. K. Saul, "Analysis and extension of spectral methods for nonlinear dimensionality reduction," in ICML '05: Proceedings of the 22nd international conference on Machine learning. New York, NY, USA: ACM Press, 2005, pp. 784-791.

[25] B. Nadler, S. Lafon, R. Coifman, and I. Kevrekidis, "Diffusion maps, spectral clustering and eigenfunctions of fokker-planck operators," in Advances in Neural Information Processing Systems 18, Y. Weiss, B. Schölkopf, and J. Platt, Eds. Cambridge, MA: MIT Press, 2006, pp. 955-962.

[26] C. M. Bachmann., T. L. Ainsworth, and R. A.Fusina, "Exploiting manifold geometry in hyperspctral imagery," IEEE Transactions on Geoscience and Remote Sensing, vol. 43, no. 3, pp. 441-454, March 2005.

[27] T. Han. and D. Goodenough, "Investigation of nonlinearity in hyperspectral remotely sensed imagery : a nonlinear time series analysis approach," IGARSS, pp. 1556-1560, July 2007.

[28] C. M. Bachmann., T. L. Ainsworth, and R. A.Fusina, "Improved manifold coordinate representations of large scale hyperspectral scenes," IEEE Transactions on Geoscience and Remote Sensing, vol. 44, no. 10, pp. 2786-2802, Octorber 2006.

[29] P. K. Robertson and J. F. O'Callaghan, "The application of perceptual color spaces to the display of remotely sensed imagery," IEEE Transactions on Geoscience and Remote Sensing, vol. 26, pp. 49-59, Jan. 1988.

[30] J. Kern and M. Pattichis, "Robust multispectral image registration using mutual-information models," IEEE Transactions on Geoscience and Remote Sensing, vol. 45, no. 5, pp. 1494-1505, May 2007.

[31] N. C. Rowe and L. L. Grewe, "Change detection for linear features in aerial photographs using edge-finding," IEEE Transactions on Geoscience and Remote Sensing, vol. 39, pp. 1608-1612, Jul. 2001.

[32] P. Shirley, M. Ashikhmin, M. Gleicher, S. Marschner, E. Reinhard, K. Sung, W. Thompson, and P. Willemsen, Fundamentals of Computer Graphics, Second Ed. Natick, MA, USA: A. K. Peters, Ltd., 2005.

[33] S. M. Pizer, E. P. Amburn, J. D. Austin, R. Cromartie, A. Geselowitz, T. Greer, B. T. H. Romeny, and J. B. Zimmerman, "Adaptive histogram equalization and its variations," Comput. Vision Graph. Image Process., vol. 39, no. 3, pp. 355-368, 1987.

[34] R. Fattal, D. Lischinski, and M. Werman, "Gradient domain high dynamic range compression," in SIGGRAPH '02: Proceedings of the 29th annual conference on Computer graphics and interactive techniques. New York, NY, USA: ACM Press, 2002, pp. 249-256.
[35] P. Heckbert, "Color image quantization for frame buffer display," SIGGRAPH Comput. Graph., vol. 16, no. 3, pp. 297-307, 1982.

[36] J. A. Hartigan and M. A. Wong, "A K-means clustering algorithm," Applied Statistics, vol. 28, pp. 100-108, 1979.

[37] T. Cox and M. Cox, Multidimensional Scaling. Chapman and Hall, 1994.

[38] V. de Silva and J. B. Tenenbaum, "Global versus local methods in nonlinear dimensionality reduction,” in NIPS, 2002, pp. 705-712.

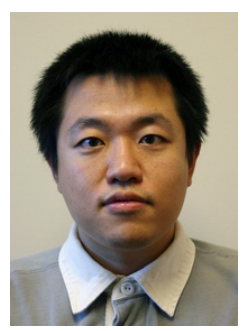

Ming Cui received his $\mathrm{BE}$ in civil engineer and MS in computer science from Zhejiang University, Hangzhou, China in 2002 and 2005 respectively. Currently he is a $\mathrm{PhD}$ candidate at Arizona State University and works in the Partnership for Research in Spatial Modeling (PRISM) Lab starting from 2005. His research interests include computer graphics and image processing.

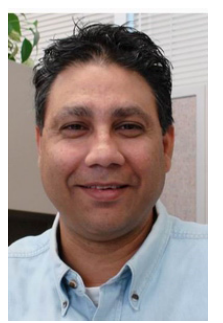

Anshuman Razdan (M'05) received the BS and MS degrees in mechanical engineering and the $\mathrm{PhD}$ degree in computer science. He is an associate professor in the Division of Computing Studies and the Director of Advanced Technology Innovation Collaboratory and the I3DEA Laboratory at Arizona State University, Polytechnic campus. He has been a pioneer in computing-based interdisciplinary collaboration and research at ASU. His research interests include geometric design, computer graphics, document exploitation, and geospatial visualization and analysis. He is the principal investigator and a collaborator on several federal grants from agencies, including the US National Science Foundation (NSF), NGA, and NIH. He is a member of the IEEE.

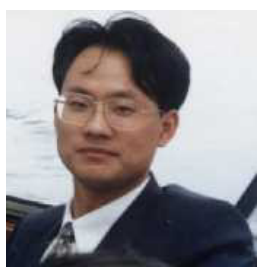

Jiuxiang Hu received the BS, MS and PhD degree in Mathematics from Huazhong Normal University in 1988, Huazhong University of Science and Technology, Wuhan, China, in 1991 and Lanzhou University, Lanzhou, China, in 1994, respectively. $\mathrm{He}$ is a research scientist in Imaging and 3D Data Exploitation and Analysis (I3DEA) lab in the Division of Computing Studies at ASU Polytech campus and Partnership for Reseach in Spatial Modeling (PRISM) Lab at Arizona State University from 2000. His research interests include computer graphics, visualization, and image processing and numerical computation. He has developed and implemented methods to segment biomedical volume data sets, including image statistical and geometric modeling and segmentation techniques with application to structural and quantitative analysis of $2 \mathrm{D} / 3 \mathrm{D}$ images.

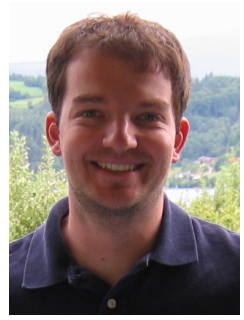

Peter Wonka (M'08) received his $\mathrm{PhD}$ and $\mathrm{MS}$ from the Technical University of Vienna in computer science. Additionally he received an MS in Urban Planning from the same institution. He is currently with Arizona State University (ASU). Prior to coming to ASU, he was a post doctoral researcher at the Georgia Institute of Technology for two years. His research interests include various topics in computer graphics, visualization, and image processing. He is a member of the IEEE. 